\title{
Context influences holistic processing of nonface objects in the composite task
}

\author{
JENNIFER J. RICHLER \\ Vanderbilt University, Nashville, Tennessee \\ Cindy M. BuKaCH \\ University of Richmond, Richmond, Virginia \\ AND \\ ISABEL GaUTHIER \\ Vanderbilt University, Nashville, Tennessee
}

\begin{abstract}
We explored whether holistic-like effects can be observed for nonface objects in novices as a result of the task context. We measured contextually induced congruency effects for novel objects (Greebles) in a sequential matching selective attention task (composite task). When format at study was blocked, congruency effects were observed for study-misaligned, but not study-aligned, conditions (Experiment 1). However, congruency effects were observed in all conditions when study formats were randomized (Experiment 2), revealing that the presence of certain trial types (study-misaligned) in an experiment can induce congruency effects. In a dual task, a congruency effect for Greebles was induced in trials in which a face was first encoded, but only when it was aligned (Experiment 3). Thus, congruency effects can be induced by context that operates at the scale of the entire experiment or within a single trial. Implications for using the composite task to measure holistic processing are discussed.
\end{abstract}

It is generally accepted that faces are processed differently from other objects (Farah, Wilson, Drain, \& Tanaka, 1998; Ge, Wang, McCleery, \& Lee, 2006; Maurer, Le Grand, \& Mondloch, 2002; Yin, 1969; but see Sekuler, Gaspar, Gold, \&, Bennett, 2004; Wenger \& Ingvalson, 2002). More specifically, face processing is believed to be holistic: Faces are processed as unified wholes rather than in terms of parts or features. A holistic processing strategy for faces is highly adaptive: Since all faces are made up of the same features in the same configuration, it is the subtle differences in the spatial relations between these features that are diagnostic of identity (Diamond \& Carey, 1986; Leder \& Bruce, 1998, 2000; Le Grand, Mondloch, Maurer, \& Brent, 2004; Mondloch, Le Grand, \& Maurer, 2002; Searcy \& Bartlett, 1996). However, one negative consequence of this holistic processing strategy is that participants are unable to selectively attend to one part of a face, even when a failure to do so negatively impacts their performance in an experimental task (e.g., Farah et al., 1998; Richler, Tanaka, Brown, \& Gauthier, 2008). Although evidence suggests that holistic processing of faces is relatively robust, here, we investigated the possibility that similar effects can also be found with nonface objects because of contextual influences. First, we describe the measure of holistic processing used in our studies, and then outline what motivated us in searching for contextually induced effects that would resemble hallmarks of holistic processing.

One paradigm that is used to assess failures of selective attention due to holistic processing is the composite task. In this task, participants are asked to match one half of a study face composite, made of the top of one face and the bottom of another, to the corresponding half of a subsequently presented test face composite. On congruent trials, both the relevant and irrelevant parts of the test face are the same as or different from the corresponding parts of the study face; on incongruent trials, one part is the same and the other part is different. Holistic processing is inferred from a congruency effect, in which performance is impaired on incongruent trials relative to congruent trials; the information in the irrelevant face half interferes with performance, despite instructions to selectively attend to the relevant half (Cheung, Richler, Palmeri, \& Gauthier, 2008; Farah et al., 1998; Gauthier, Curran, Curby, \& Collins, 2003; Richler, Gauthier, Wenger, \& Palmeri, 2008; Richler, Tanaka, et al., 2008). Importantly, the congruency effect is larger for faces than for other objects (Farah et al., 1998) and increases with perceptual expertise (Gauthier \& Tarr, 2002).

Often, studies using the composite task also use an alignment manipulation. The standard finding is that the congruency effect is reduced when the face halves are

J. J. Richler, jennifer.j.richler@vanderbilt.edu 
misaligned at test; when the face halves are no longer presented in the meaningful arrangement, holistic processing is attenuated (Cheung et al., 2008; Richler, Gauthier, et al., 2008; Richler, Tanaka, et al., 2008). In a recent study, the arrangement of face halves was also manipulated at study, and it was found that the magnitude of the congruency effect was unaffected by whether the study face was aligned or misaligned (Richler, Tanaka, et al., 2008).

The holistic effects measured with the composite task are generally not found with familiar objects (Robbins \& McKone, 2007) or novel objects (Gauthier \& Tarr, 2002) in novices. However, these studies with objects used a version of the composite task in which the irrelevant halves of the composites were always different. In this socalled partial design, same trials are always incongruent, whereas different trials are always congruent, and holistic processing is inferred from an alignment effect that is unrelated to congruency. Although this partial variant of the composite task has also been used with faces (e.g., Goffaux \& Rossion, 2006; Hole, 1994; Le Grand et al., 2004; Michel, Rossion, Han, Chung, \& Caldara, 2006; Young, Hellawell, \& Hay, 1987), recent work has shown that it comes with serious limitations (see Cheung et al., 2008; Gauthier \& Bukach, 2007). Critically, in this version of the task, only performance on same trials is considered. Thus, differences in response bias between aligned and misaligned trials (see Cheung et al., 2008) could be misinterpreted as true discriminability differences. Moreover, same trials are always incongruent, and congruency itself has been shown to affect response biases as well (Cheung et al., 2008; Farah et al., 1998).

In only one study using objects has the variant of the composite task described earlier, in which holistic processing is measured in terms of congruency effects, been used. It was found in this study that although car experts showed a larger congruency effect than did car novices, consistent with the suggestion that holistic processing emerges with perceptual expertise (Gauthier \& Tarr, 2002), car novices did show a small congruency effect (Gauthier et al., 2003). This may not be surprising, because the composite task is similar to other selective attention paradigms, such as the Stroop task: On incongruent trials, participants need to ignore information that leads to a conflicting response (MacLeod, 1991). Indeed, with faces, the congruency effect has been mainly attributed to interference on incongruent trials (Richler, Tanaka, et al., 2008). Moreover, the car novices in Gauthier et al. (2003) would still have had some visual experience with cars, albeit not to the same extent as the car experts. Perhaps the small congruency effect observed for novices in this case reflected this basic knowledge and experience. Thus, the goal of the present work was to examine congruency effects for novel objects in true novices, using completely novel objects.

\section{EXPERIMENT 1}

\section{Method}

Participants. Forty-two participants completed the experiment. Twenty-one were assigned to each condition (study aligned vs. study misaligned). One participant's data were discarded from the studyaligned condition because of a failure to respond on more than $10 \%$ of the trials, and 2 more participants' data ( 1 from each condition) were discarded for below-chance performance.

Stimuli. Stimuli were made from images of 16 asymmetrical computer-generated novel objects (Greebles), made up of 4 Greebles from four different families (the families are defined by common central shapes). Asymmetrical Greebles (Rossion, Kung, \& Tarr, 2004) were created by transforming all of an original group of symmetrical Greebles (Gauthier \& Tarr, 1997) in the same manner, to produce asymmetrical configurations of parts common to all objects (see Figure 1). Greebles were divided into top and bottom parts, which were combined to make 32 composites. Tops and bottoms were always combined within the same family. Each composite Greeble was approximately $200 \times 160$ pixels in size. A black line 3 pixels thick separated top and bottom halves.

Misaligned Greebles were made by moving the bottom parts of each composite 70 pixels to the right, so that the edge of the bottom half of the Greeble fell on the center of the top half.

Procedure. The experiment was conducted on Mac OS 9 computers (19-in. monitor, 1,280 × 960 pixel resolution) using RSVP software (Williams \& Tarr, n.d.). Participants were seated approximately $60 \mathrm{~cm}$ from the monitor, although head position was not fixed. On each trial, a study stimulus was shown for $700 \mathrm{msec}$, followed by a flashing mask (four identical random pattern masks shown for $120 \mathrm{msec}$ each, alternating with a 50-msec blank screen, for a total of $630 \mathrm{msec}$ ). Participants assigned to the study-aligned condition saw an aligned Greeble composite as the study stimulus, whereas participants assigned to the study-misaligned condition always saw a misaligned Greeble composite as the study stimulus. Next, a rectangular bracket that cued either a top or a bottom judgment was shown for $300 \mathrm{msec}$ and remained on the screen when the test stimulus appeared. The test stimulus was displayed until the participant responded, or for $4,000 \mathrm{msec}$ if no response was given. Participants were instructed to indicate by buttonpress whether the cued part was the same or different at study and test. Participants were instructed to attend to both parts of the study stimulus and to

\section{Test Greeble}

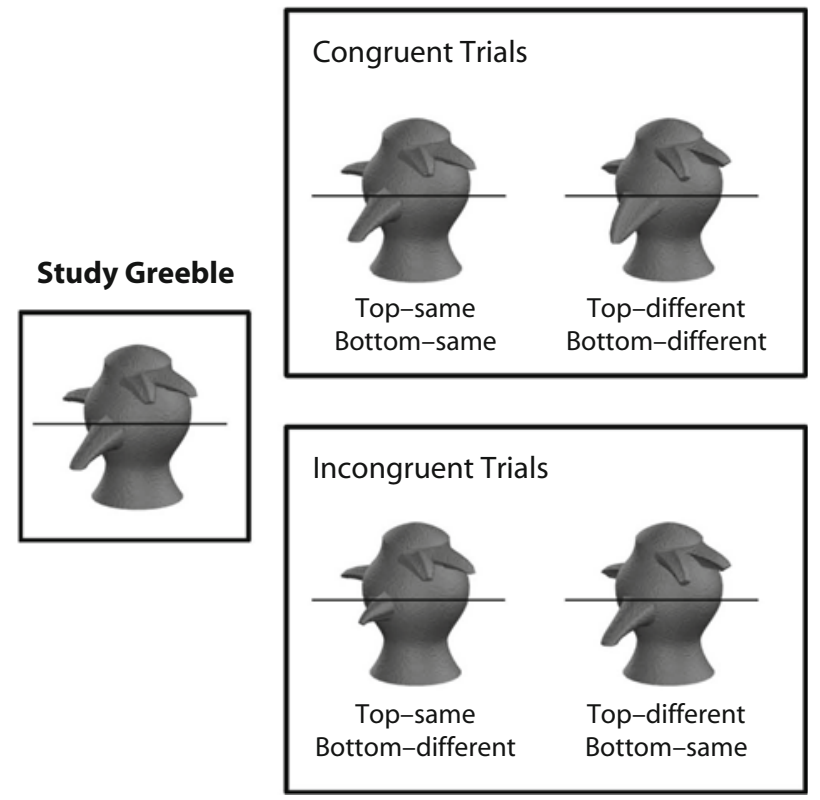

Figure 1. Illustration of the trial types in the composite task. On congruent trials, both the top and bottom of the test Greeble are the same as or different from the corresponding parts of the study Greeble. On incongruent trials, one part is the same as and the other part is different from the corresponding parts of the study Greeble. 
ignore the uncued part (if any) of the test stimulus. No feedback was given.

There were 240 experimental trials. On 48 trials, an isolated part was shown at test (12 trials for each combination of top/bottom and same/different). The remaining trials (192) included 12 trials in each combination of test configuration (aligned vs. misaligned), congruency (congruent vs. incongruent), cued part (top vs. bottom), and correct response (same vs. different). Trial types were randomized for each participant. The experimental trials were preceded by 10 practice trials.

\section{Results}

Sensitivity $\left[d^{\prime}=z\right.$ (hit) $-z(\mathrm{FA})$, calculated for each participant (FA; false alarm); see Macmillan \& Creelman, 2005, for a discussion of selecting appropriate sensitivity measures $]$, criterion $\{c=-1 / 2[z$ (hit $)-z(\mathrm{FA})]\}$, and hit and false alarm rates for congruent and incongruent trials when the study and test Greebles were aligned or misaligned are plotted in Figure 2. Although significant response biases are frequently observed in composite tasks

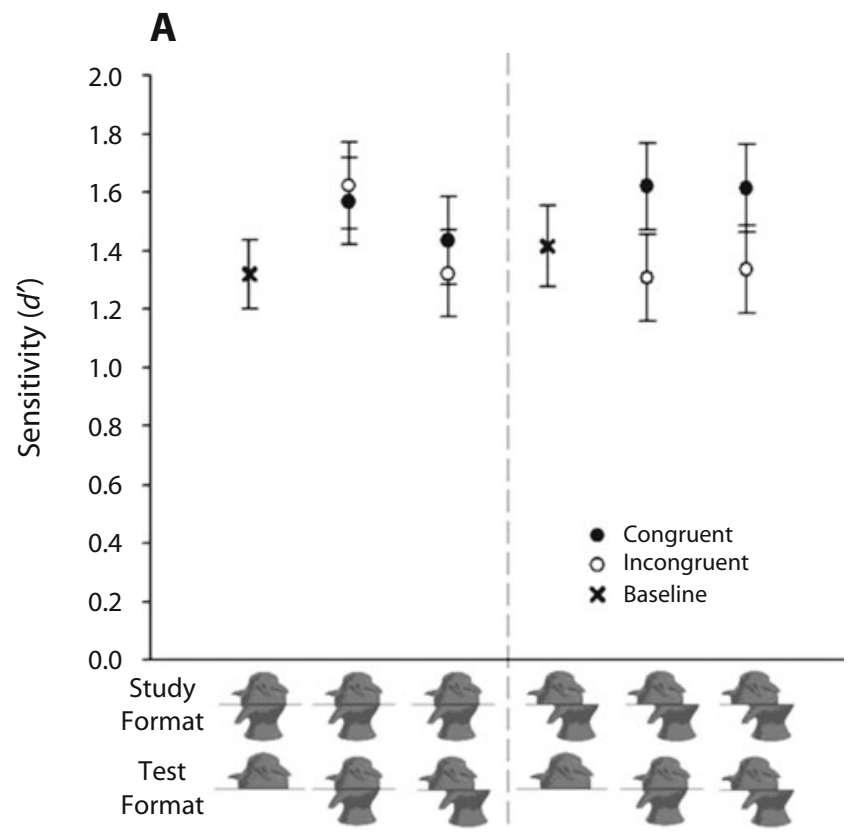

B

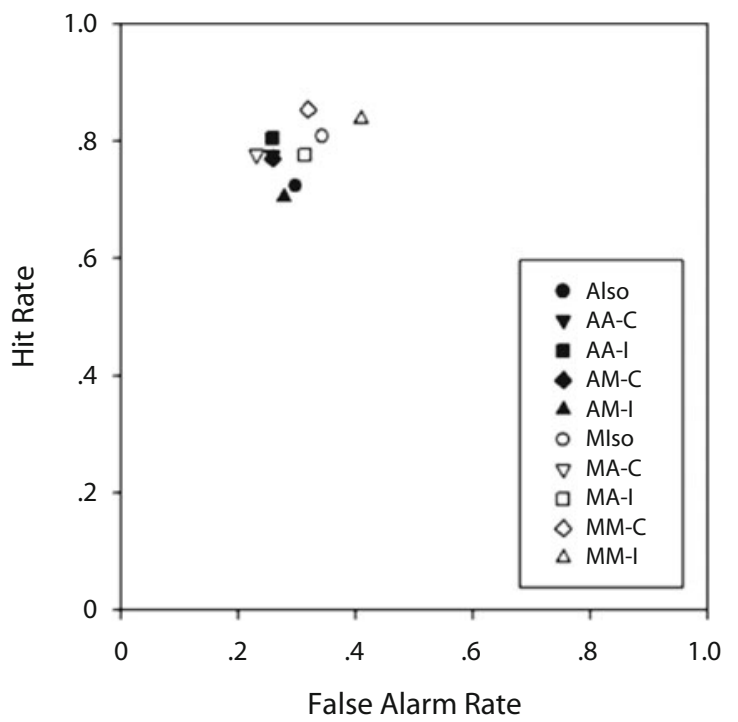

C

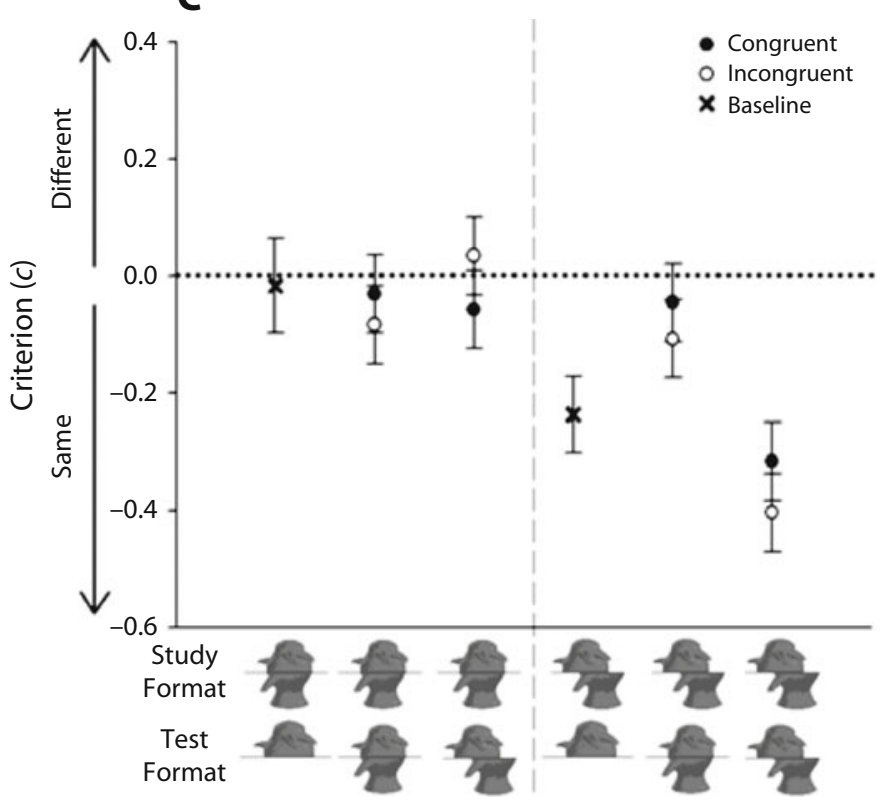

Figure 2. (A) Sensitivity ( $\left.d^{\prime}\right)$, (B) hit and false alarm rates, and (C) criterion (c) for congruent and incongruent trials in Experiment 1, for all combinations of aligned and misaligned study and test Greebles. Study format was blocked between participants. The legend for Figure 2B shows study format (A, aligned; M, misaligned), test format (A, aligned; M, misaligned; Iso, isolated), and congruency (C, congruent; I, incongruent). Error bars show $95 \%$ confidence intervals for within-subjects effects for all data points, except in the isolated baseline conditions, where error bars show standard errors of the means. 
with faces (e.g., Cheung et al., 2008; Richler, Gauthier, et al., 2008; Richler, Tanaka, et al., 2008), there is not yet a comprehensive account of how these biases should be interpreted. As such, although we report in full the analyses on response bias, discussion of these data is limited.

Sensitivity $\left(\boldsymbol{d}^{\prime}\right)$. As can be appreciated from Figure 2A, congruency effects were only observed when the study Greeble was misaligned and were not affected by the format of the test Greeble.

These observations are confirmed by inferential statistics. A $2 \times 2 \times 2$ mixed-factors ANOVA was conducted on sensitivity $\left(d^{\prime}\right)$ with test Greeble format (aligned vs. misaligned) and congruency (congruent vs. incongruent) as repeated measures factors and study Greeble format (aligned vs. misaligned) as a between-subjects factor. There was a significant main effect of congruency, such that performance was better on congruent trials than on incongruent trials $\left[F(1,37)=6.999, M S_{\mathrm{e}}=.147, p<.05\right]$. Critically, there was a significant congruency $\times$ study Greeble format interaction $\left[F(1,37)=4.701, M S_{\mathrm{e}}=.147\right.$, $p<.05]$, such that there was only a congruency effect when the study Greeble was misaligned $[F(1,18)=5.182$, $\left.M S_{\mathrm{e}}=.174, p<.05\right]$. As can be seen in Figure 2B, congruency effects were driven by an increase in false alarms on incongruent trials relative to congruent trials.

Bonferroni-corrected paired-sample $t$ tests $(\alpha=.00625)$ for each condition with its test-isolated baseline revealed no significant facilitation or interference.

Criterion (c). A $2 \times 2 \times 2$ mixed-factors ANOVA on criterion (see Figure 2C) revealed a significant main effect of test format $\left[F(1,37)=7.057, M S_{\mathrm{e}}=.078, p<.05\right]$ that was modulated by an interaction with study format $\left[F(1,37)=13.599, M S_{\mathrm{e}}=.078, p<.01\right]$, such that participants were more likely to respond "same" when both the study and test formats were misaligned.

\section{Discussion}

In Experiment 1, we showed that it is possible to see a congruency effect with a nonface novice category; however, this effect was observed only when the study Greeble was misaligned, suggesting that the arrangement of parts at study is critical for producing a congruency effect with a completely unfamiliar object category. This is in contrast to what has been observed with faces in the composite task, where the arrangement of parts at test is critical for producing congruency effects, regardless of the arrangement of parts at study (Richler, Tanaka, et al., 2008).

In Experiment 1, study Greeble formats were manipulated between participants. Therefore, it is unknown whether the context created by the study-misaligned trials operated within a single trial or over the entire experiment. In Experiment 2, study Greeble formats were randomized within subjects. If the study-misaligned Greebles induce a context for a single trial, we would expect to see congruency effects only for study-misaligned trials and not for study-aligned trials. If, on the other hand, the mere presence of the study-misaligned trials causes a change in context that affects the entire experiment, we would expect to see congruency effects in both study conditions when these trial types are randomized.

\section{EXPERIMENT 2}

\section{Method}

Participants. Thirty-seven participants completed the experiment. Data from 6 participants were discarded because of belowchance performance.

Stimuli. The stimuli were the same as those in Experiment 1.

Procedure. Each trial unfolded in the same manner as did those in Experiment 1.

There were 480 experimental trials. On 96 trials, an isolated part was shown at test (12 trials for each combination of study aligned/ misaligned, same/different, and top/bottom). The remaining trials (382) included 12 trials in each combination of study configuration (aligned vs. misaligned), test configuration (aligned vs. misaligned), congruency (congruent vs. incongruent), cued part (top vs. bottom), and correct response (same vs. different). Trial types were randomized for each participant. Twelve practice trials preceded the experimental trials.

\section{Results}

Sensitivity $\left(d^{\prime}\right)$, criterion $(c)$, and hit and false alarm rates on congruent and incongruent trials as a function of study and test Greeble configuration are plotted in Figure 3.

Sensitivity $\left(d^{\prime}\right)$. As can be appreciated from Figure 3A, a congruency effect was observed in all conditions.

This observation was confirmed by a $2 \times 2 \times 2$ repeated measures ANOVA with study Greeble format (aligned vs. misaligned), test Greeble format (aligned vs. misaligned), and congruency (congruent vs. incongruent) as factors. There was a significant main effect of congruency, such that performance was greater on congruent trials than on incongruent trials $\left[F(1,30)=14.701, M S_{\mathrm{e}}=1.138, p<\right.$ $.01]$. Critically, congruency did not interact with either test or study format, indicating comparable congruency effects across all conditions. As can be seen in Figure 3B, congruency effects arose from a decrease in hits as well as an increase in false alarms on incongruent trials relative to congruent trials.

There was a significant study format $\times$ test format interaction $\left[F(1,30)=5.770, M S_{\mathrm{e}}=.122, p<.05\right]$, such that the average performance was better when both the study and test Greebles were aligned than when the study Greeble was misaligned and the test Greeble was aligned $\left[F(1,30)=6.513, M S_{\mathrm{e}}=.107, p<.05\right]$.

Bonferroni-corrected paired-sample $t$ tests $(\alpha=.00625)$ for each condition with its test-isolated baseline revealed no significant facilitation or interference. Because congruency effects can arise only from facilitation and/or interference, this pattern was likely obtained because of small amounts of both interference and facilitation.

Criterion (c). A $2 \times 2 \times 2$ repeated measures ANOVA on criterion revealed a significant main effect of study format $\left[F(1,36)=7.035, M S_{\mathrm{e}}=.056, p<.05\right]$ that was modulated by an interaction with test format $[F(1,36)=$ $\left.35.474, M S_{\mathrm{e}}=.068, p<.01\right]$, such that participants were more likely to respond "same" when the study and test formats matched (i.e., study and test both aligned or study and test both misaligned; see Figure 3C). The three-way interaction between study format, test format, and congruency was also significant $\left[F(1,36)=9.709, M S_{\mathrm{e}}=.037\right.$, $p<.01]$, such that participants were more likely to respond 


\section{A}

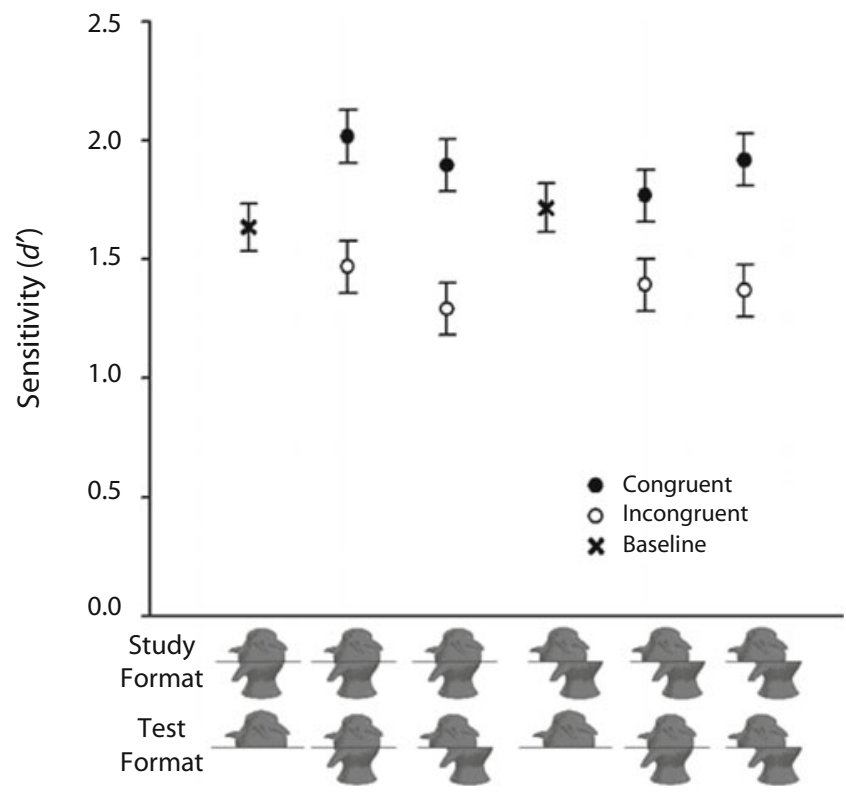

B

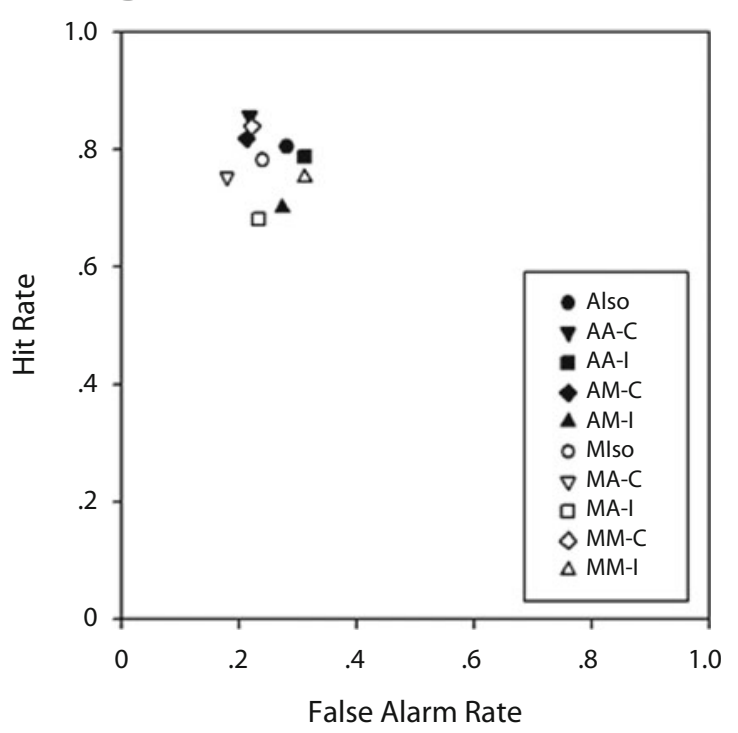

C

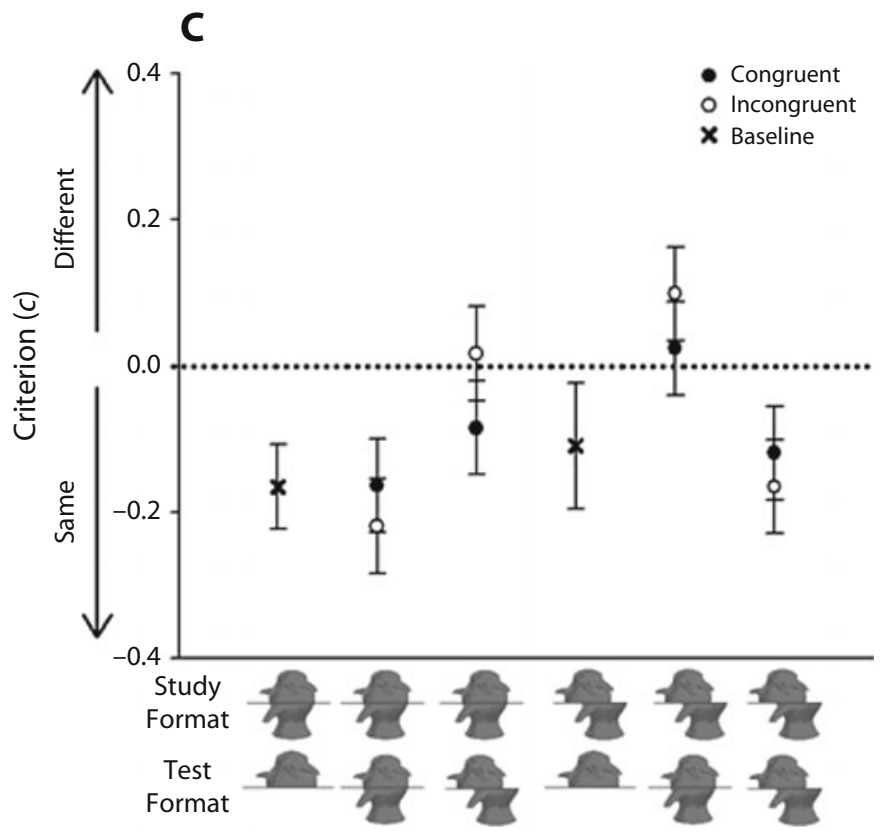

Figure 3. (A) Sensitivity $\left(d^{\prime}\right)$, (B) hit and false alarm rates, and (C) criterion (c) for congruent and incongruent trials in Experiment 2 , for all combinations of aligned and misaligned study and test Greebles. Study format was randomized for all participants. The legend for Figure 3B shows study format (A, aligned; M, misaligned), test format (A, aligned; M, misaligned; Iso, isolated), and congruency (C, congruent; I, incongruent). Error bars show $95 \%$ confidence intervals for within-subjects effects for all data points, except in the isolated baseline conditions, where error bars show standard errors of the means.

"same" on incongruent trials when study and test formats matched and more likely to respond "same" on congruent trials when study and test formats did not match.

\section{Discussion}

In Experiment 1, congruency effects were observed only for misaligned study items when study conditions were blocked. However, when study formats were randomized within the experiment in Experiment 2, equivalent congru- ency effects were observed in all conditions. This result suggests that misaligned study items do not simply influence processing of the test item within the context of a single trial but, rather, induce a context that influences all trials in the experiment. Thus, we have shown that whether stimulus conditions are blocked or randomized changes the context of the experiment, and these different experimental contexts can affect whether congruency effects are observed. 
In Experiments 1 and 2, we showed that contexts created by a misaligned study Greeble can influence congruency effects for Greebles in novices. One question is whether contextual influences can occur across object categories. For example, faces are processed more holistically than nonface objects, and this occurs automatically, without any inducing context and despite instructions to attend selectively to parts. Could processing faces within an experiment create a context that induces congruency effects for Greebles? Indeed, aligned face and car stimuli were interleaved in Gauthier et al. (2003). Perhaps car novices showed small congruency effects for cars because of the context of the experiment created by the presence of the face stimuli.

Several studies have shown that engaging a local or global processing strategy in one task can influence processing in a subsequent task. Macrae and Lewis (2002) had participants perform a letter identification task with Navon letters (Navon, 1977), which are large letters made up of smaller letters (e.g., an X composed of Ys). Half of the participants were asked to identify the large letter (i.e., a global processing task), and half of the participants were asked to identify the small letters (i.e., a local processing task). The results showed that, relative to a control group, face recognition performance was impaired when participants completed the local processing task, and face recognition performance was enhanced when participants completed the global processing task (see also Perfect, 2003). A similar contextual effect has also been observed for recognition performance for halves of composite faces (Weston \& Perfect, 2005). In
Ge et al. (2006), simultaneous matching of ambiguous figures (similar to both faces and Chinese characters) led to an inversion effect only when primed with a different task with faces and not Chinese characters. These results suggest that the context prior to which a recognition or matching task is completed can affect performance by inducing a certain processing strategy. However, the temporal dynamics of these effects are not known (whether, for instance, context could vary from one trial to another).

In Experiment 3, we examined whether congruency effects for Greebles could be contextually induced across object categories within a single trial. We used a dual task in which a face composite task and a Greeble composite task were interleaved (see Figure 4). Would the processing style for the study face-which appeared before the test Greeble - affect processing of the test Greeble and induce congruency effects? Critically, the study face was aligned or misaligned. Aligned faces are processed more holistically than misaligned faces (Cheung et al., 2008; Richler, Gauthier, et al., 2008; Young et al., 1987). Thus, we predicted that the trial context in the aligned-face condition should induce more of a congruency effect for interleaved Greeble trials than the misaligned-face condition would.

\section{EXPERIMENT 3}

\section{Method}

Participants. Twenty participants completed this study in exchange for course credit or $\$ 6$. Data from 2 participants were discarded because of below-chance performance on the Greeble task.

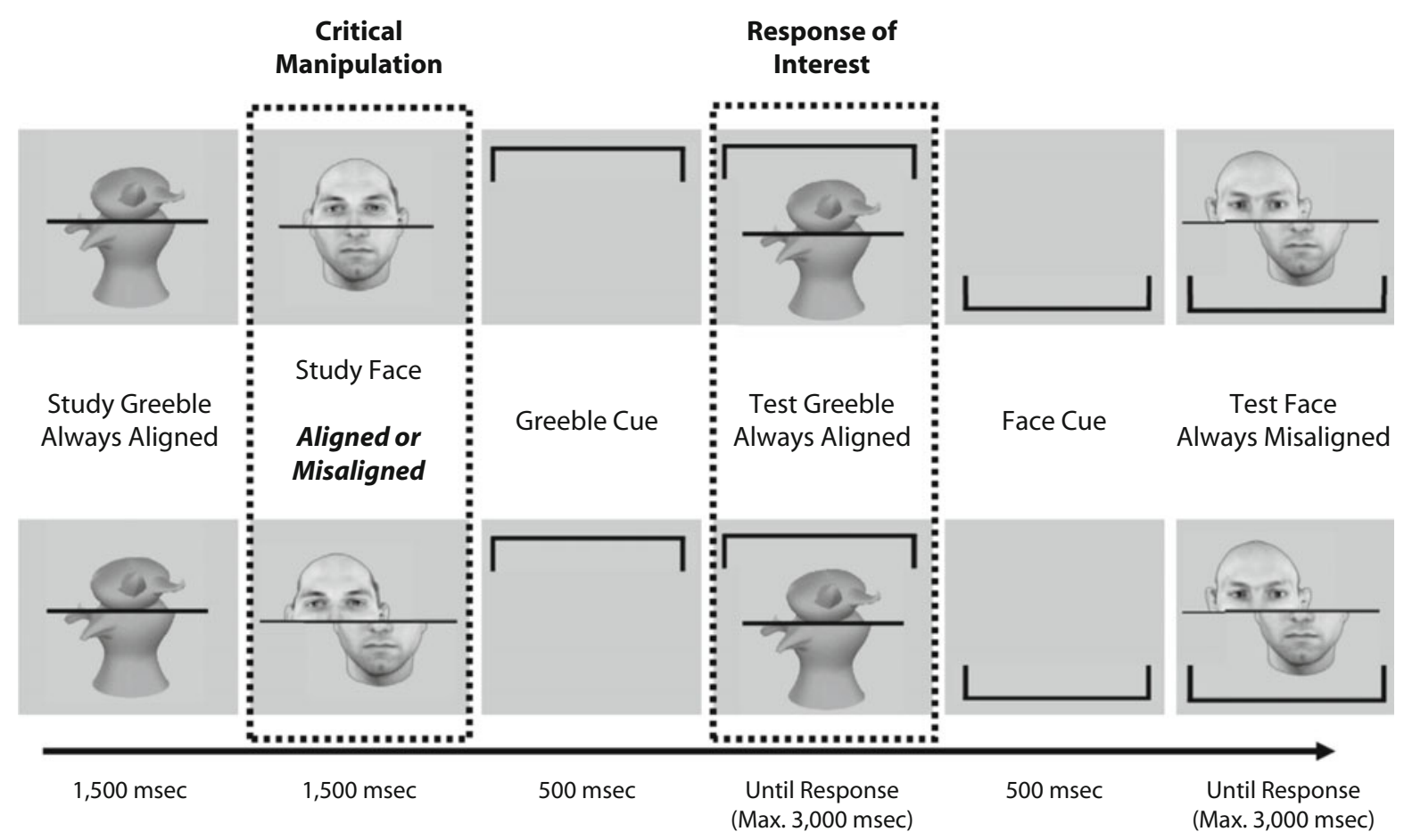

Figure 4. Schematic of two trials in the interleaved composite task used in Experiment 3. A composite task with Greebles, in which the study and test Greebles were always aligned, was interleaved with a composite task with faces, in which the study face was either aligned or misaligned and the test face was always misaligned. The critical manipulation was whether the face that preceded the test Greeble was aligned or misaligned. The response of interest was the response to the test Greeble: Would this be affected by whether the preceding face was aligned or misaligned? 
Stimuli. Greeble stimuli were made in the same manner as those in Experiment 1.

Face stimuli were created from 12 digital images of similar male faces taken from the face database developed by the Max Planck Institute for Biological Cybernetics in Tübingen, Germany (Troje \& Bülthoff, 1996). These faces did not have hair, beards, or other salient diagnostic features. Each face was approximately $200 \times 160$ pixels in size and was converted to grayscale. Faces were divided into top and bottom halves, which were reorganized to create 24 composite faces. A black line 3 pixels thick separated the two face parts.

Misaligned face composites were created by moving the bottom parts of each composite 70 pixels to the right, so that the edge of the bottom half of the face fell on the center of the top half.

Procedure. Two composite tasks - one with Greebles and one with faces - were interleaved (see Figure 4). Participants were instructed to judge whether the cued part of the test stimulus was the same as or different from the corresponding part of the study stimulus, while ignoring the irrelevant part.

At the beginning of each trial, the study Greeble was presented for $1,500 \mathrm{msec}$, followed by the study face, which was presented for $1,500 \mathrm{msec}$. We refer to the study face as the inducing face, because we are interested in whether it can create a context that will lead to congruency effects for Greebles. The inducing face was either aligned or misaligned. Following the inducing face, a square bracket was presented for $500 \mathrm{msec}$, cuing participants as to which part of the test Greeble they would be asked to respond to, followed by the test Greeble, which remained on the screen for a maximum of 3,000 msec or until participants made a response. Then another square bracket, cuing which part of the face they would have to respond to, was presented for $500 \mathrm{msec}$, followed by the test face, which remained on the screen for a maximum of $3,000 \mathrm{msec}$ or until a response was made.

Several things were kept constant in the experiment. The study and test Greebles were always aligned, because this condition did not lead to congruency effects in Experiment 1; if we saw congruency effects for Greebles in this experiment, this would ensure that they depended on the context created by the preceding face, not on the format of the study and test Greebles. The test face was always misaligned, because we did not want an aligned face at test to potentially induce holistic processing for the study Greeble on the next trial. These factors resulted in a situation in which we expected no measurable difference in holistic processing for the aligned and misaligned study face conditions, because these conditions result in comparable congruency effects at test when the test face is misaligned (Richler, Tanaka, et al., 2008). Nonetheless, we predicted that aligned and misaligned study faces would be perceived differently, and any difference obtained with Greebles between the two face contexts would be evidence to that effect. Trials on which the inducing face was aligned or misaligned were randomized, because we wanted to detect effects that could occur within a trial; if trials were blocked, we would not know whether the effect occurred within the trial or was due to a general strategic effect of context, as we saw in Experiment 2. Finally, the study Greeble was always presented first, so that only the study face would be seen before the test Greeble; if the study face had been presented first, both the study and test faces would have been presented before the test Greeble, so it would be unclear what effect the alignment of the study face had on the congruency effect for the test Greeble. This design would not have allowed us to determine whether face processing creates a context effect only when a face is preceded by an object. We aimed to create conditions that would be sufficient to produce a context effect but could not address whether they were necessary.

There were 192 experimental trials, with 12 trials for each combination of cued part (top vs. bottom), congruency (congruent vs. incongruent), correct response (same vs. different), and inducing face format (aligned vs. misaligned) for the Greeble composite task. A practice block of 4 trials preceded the experimental block. For the face task, there were 12 trials for each combination of part, congruency, correct response, and study face format for the Greeble com- posite task, and each trial type was randomly paired with a face trial type (in terms of congruency and correct response). Face trial types were randomly paired with Greeble trial types.

\section{Results}

As predicted, and replicating prior results (Richler, Tanaka, et al., 2008), there was no difference in the congruency effect for misaligned test faces obtained in trials with aligned or misaligned study faces (alignment $\times$ congruent interaction, $p=.975$ ). However, we nonetheless found evidence that aligned and misaligned faces are processed differently, in that they produce different contexts for the Greeble composite task. Sensitivity $\left(d^{\prime}\right)$, criterion $(c)$, and hit and false alarm rates for congruent and incongruent trials for the Greeble composite task, as a function of whether the inducing face was aligned or misaligned, are plotted in Figure 5.

Sensitivity $\left(d^{\prime}\right)$. As can be appreciated from Figure 5A, a congruency effect for Greebles was observed only when the test Greeble was preceded by an aligned face.

A $2 \times 2$ repeated measures ANOVA with congruency (congruent vs. incongruent) and format of the inducing face (aligned vs. misaligned) as factors revealed a significant main effect of congruency, with better performance on congruent than on incongruent trials $[F(1,17)=7.596$, $\left.M S_{\mathrm{e}}=.110, p<.05\right]$. Critically, there was a congruency $\times$ face format interaction $\left[F(1,17)=4.496, M S_{\mathrm{e}}=.088, p<\right.$ $.05]$. Post hoc $t$ tests (Bonferroni corrected for multiple comparisons, $\alpha=.025$ ) revealed that there was a congruency effect only when the inducing face was aligned, and, as can be seen in Figure 5B, the congruency effect was driven by a decrease in hits and an increase in false alarms on incongruent trials relative to congruent trials.

Criterion $(\boldsymbol{c})$. A $2 \times 2$ repeated measures ANOVA revealed no significant main effects or interactions (see Figure 5C).

\section{Discussion}

In Experiment 3, we found that contextually induced congruency effects can occur within a trial when the context is created by an object from a different category. Specifically, trials that contained aligned faces led to congruency effects for Greebles. One explanation of this finding is that it results from hysteresis in terms of the processing strategy engaged by the inducing face: Because participants have perceived the aligned face holistically, they also process the following Greeble more holistically. This could occur because of a cost in switching between holistic and analytical strategies (Hübner, 2000; Hübner, Futterer, \& Steinhauser, 2001). Although the context effect must be caused by the change in face alignment (the only difference between conditions), it is possible that the effect depends on an interaction with the Greeble that is always presented at trial outset (for instance, a certain load in visual short-term memory could be necessary). Further experiments could investigate the necessary and sufficient conditions for this contextual effect. However, here, we were primarily concerned with the demonstration that encoding of aligned and misaligned faces produces different contexts that influence congruency effects for 
A

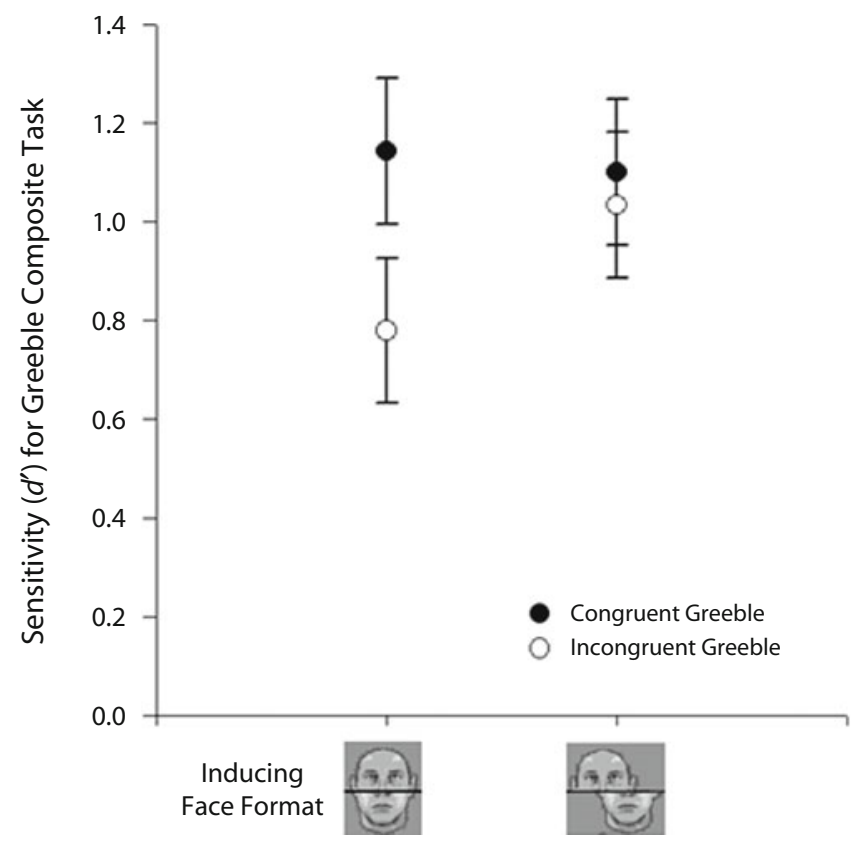

B

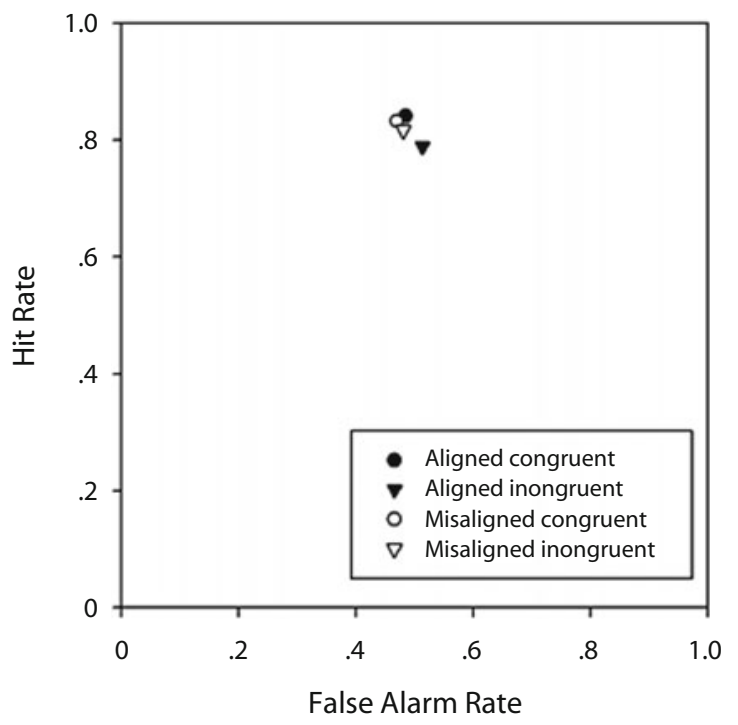

C

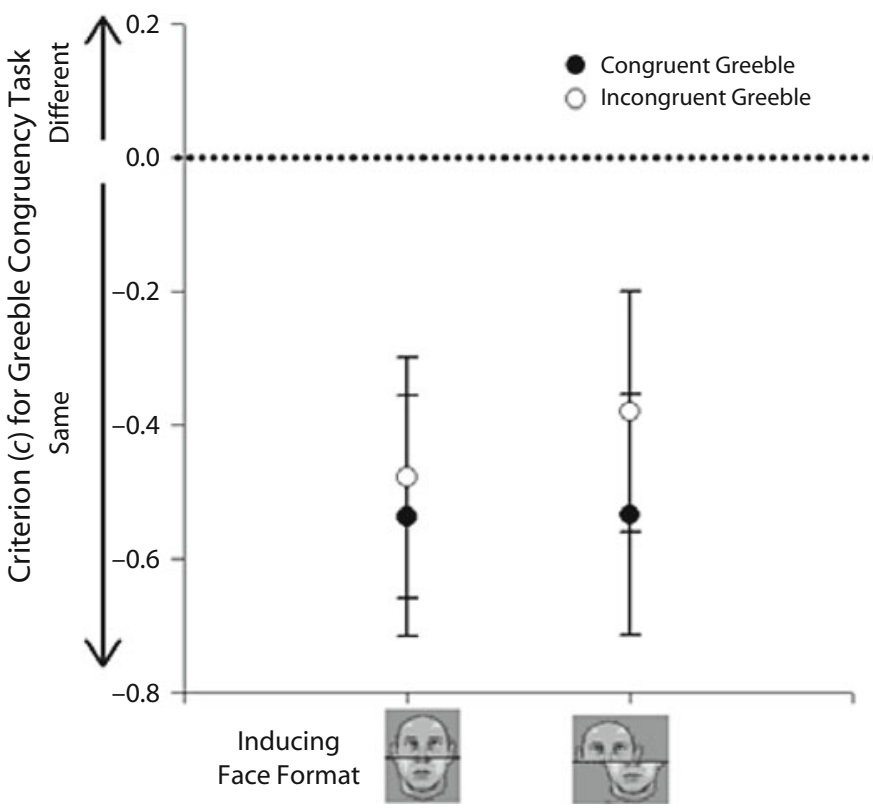

Figure 5. (A) Sensitivity $\left(d^{\prime}\right)$, (B) hit and false alarm rates, and (C) criterion (c) on the Greeble composite task in Experiment 3 when the Greeble trial was congruent and incongruent, as a function of whether the preceding face was aligned or misaligned. The legend for Figure 5B lists whether the inducing face was aligned or misaligned and whether the Greeble was congruent or incongruent. Error bars show $95 \%$ confidence intervals of within-subjects effects.

novel objects, with the context rapidly changing from one trial to the next.

Indeed, there are several reports in which processing style recruited by one task has influenced processing on a subsequent task (e.g., Macrae \& Lewis, 2002; Perfect, 2003; Weston \& Perfect, 2005; but see Lawson, 2007) or in which presentation of an unambiguous stimulus has influenced the interpretation and processing of similar shapes that are more ambiguous (Bentin, Sagiv, Mecklinger, Friederici, \& von Cramon, 2002; Ge et al., 2006). In addition to revealing contextual effects at the scale of single trials, our findings extend this prior work, because, here, context is not created by requiring participants to first perform a task with a specific strategy. Moreover, 
the processing of faces influences that of asymmetrical Greebles, despite clear differences in the geometries of the two types of stimuli.

In Experiments 1 and 2, we suggested that misaligned study Greebles led to congruency effects because those Greebles must influence the strategy with which participants process other objects in the experiments. Although misaligned Greebles are arguably perceptually more similar to misaligned faces than to aligned faces, it was nonetheless the aligned faces that induced a congruency effect in Experiment 3. However, there were several differences between the tasks used in Experiments 1 and 2 and the task in Experiment 3, such as differences in timing and working memory demands. These results are consistent with the notion that the contextual effects are not perceptual (as in Ge et al., 2006) but rather are strategic in nature.

\section{GENERAL DISCUSSION}

In Experiment 1, when study formats were blocked, a congruency effect was observed for novel objects (Greebles), but only when the study Greeble was misaligned. In Experiment 2, when study formats were randomized, congruency effects for Greebles were observed in all conditions of the experiment, irrespective of study format. These results suggest both that the presence of certain trial types can create a context that leads to congruency effects throughout an experiment and that changing the experimental context by blocking rather than randomizing conditions can change whether congruency effects are observed. In Experiment 3, we found that contextual effects can occur across object categories and within a trial.

Together, our results suggest that congruency effects can be influenced by context. It is interesting to speculate why faces and objects ${ }^{1}$ may have induced context effects on different temporal scales. Misaligned Greebles induced congruency effects on the scale of the whole experiment (Experiments 1 and 2); aligned faces induced congruency effects across categories within the context of a single trial (Experiment 3 ). One important difference between aligned faces and misaligned objects is that faces are processed holistically in an automatic fashion; that is, the effect occurs naturally outside of any inducing context and despite instructions to attend to face parts. In contrast, misaligned objects are not processed holistically, and, in fact, it may require considerable effort to encode both parts of a complex object and to bind them despite interference from similar objects in the same experiment. It is possible that effortful strategies are more long lasting because of the likely costs of engaging and inhibiting them. In contrast, a perceptual strategy that is more automatic - such as holistic processing of faces - may have much faster temporal dynamics. In our experiments, it is possible that faces influenced Greebles only because of the need to keep the inducing face in working memory during the Greeble decision. Clearly, future work is required in order to uncover the mechanism underlying these two kinds of contextual effects and to compare them with other similar types of influences in the literature. What is critical here is the fact that different contexts can lead to different congruency effects that would not be predicted if congruency effects arose solely because of holistic processing.

Thus, our findings have important implications for measures of holistic processing. Holistic processing in the version of the composite task used here is measured in terms of congruency: Participants cannot selectively attend and so are affected by information in the irrelevant half of the image. Although it could be argued that congruency effects arise because of response conflict, analogous to the effects seen in the Stroop literature (MacLeod, 1991), recent work has suggested that this is not the case (Richler, Cheung, Wong, \& Gauthier, in press). Moreover, response conflict by itself would not account for the fact that congruency effects are larger for faces than for other objects (Farah et al., 1998; Gauthier et al., 2003) and increase with expertise for objects (Gauthier et al., 2003; Gauthier \& Tarr, 2002). Consistent with these reports, outside of an inducing context, we found no holistic processing in our experiments for Greebles in novices (when study format was aligned in a blocked design).

However, in Experiments 1 and 2, we were able to show that congruency effects for novel objects in novices arise in certain contexts, such as when study items are misaligned and when misaligned study items are randomized with aligned study items. Is it possible to distinguish between congruency effects that are due to contextual strategy and those that arise because of holistic processing as a result of expertise? In studies with faces, the congruency effect is modulated by the alignment of the test face (Cheung et al., 2008; Richler, Gauthier, et al., 2008; Richler, Tanaka, et al., 2008) and mainly consists of interference on incongruent trials (Richler, Tanaka, et al., 2008). For novel objects in Experiments 1 and 2, there was no significant interference with respect to baseline, and alignment at test did not impact the congruency effect. This result suggests that although congruency effects for faces and objects of expertise are larger than the effects presented here, the congruency effect on its own may not be a sufficient index of face-like holistic processing. Rather, the comparisons of congruent and incongruent trials with a baseline and the interaction between congruency and alignment may be more indicative of holistic processing driven by the stimulus, as opposed to congruency effects that arise because of the context of the task. Indeed, once participants become experts at individuating objects from a novel category, they show a congruency effect that is modulated by alignment, just as is found with faces (Wong, Palmeri, \& Gauthier, in press).

The contextual influences on the congruency effect described in the present work with novel objects could equally well affect the congruency effects observed for faces. However, and perhaps more important, the distinction between congruency effects that arise because of holistic processing and contextually induced congruency effects may be useful in understanding some recent results obtained with faces. For example, in Experiment 3 of Richler, Tanaka, et al. (2008), the cue indicating which part participants needed to respond to was presented after the presentation of the test face (unlike in most experiments using the composite task, in which the cue is pre- 
sented either before or simultaneously with the test face). Under these conditions, a congruency effect that was not modulated by the alignment of the test face was observed following misaligned study faces when study format was blocked. It may be that, in these conditions, congruency effects were due to contextually induced strategies, as opposed to a holistic processing mechanism specific to faces and other objects of expertise. A better understanding of the conditions that lead to contextual congruency effects, rather than to holistic congruency effects, with faces is crucial to appreciating how processing differs between faces and objects of expertise and common objects.

Recent work with individuals with autism may also benefit from an understanding of contextually induced congruency effects. Deficits in face processing are well documented in autism; however, whether these deficits are due to a deficit in holistic processing is still a source of debate. A recent study found that although individuals with autism show a congruency effect for faces, this congruency effect is not affected by misalignment at test (Gauthier, Klaiman, \& Schultz, in press). Critically, in this experiment, study items were aligned or misaligned, and these conditions were randomized. Thus, congruency effects for faces in individuals with autism may have been observed because of context, analogous to the congruency effects observed with novel Greebles in Experiment 2, as opposed to arising because of holistic processing. This would be consistent with the notion that individuals with autism process faces like typical participants process objects that they are not experts with. Although this interpretation remains to be tested, it illustrates the usefulness of a framework that can distinguish face-like and contextual congruency effects.

There are many factors that potentially contribute to performance in the composite task, including possible strategy shifts for different experimental contexts (e.g., blocked vs. randomized design), as demonstrated here, and response biases (e.g., Cheung et al., 2008), which have nothing to do with whether the stimuli are being processed holistically. Indeed, in Experiments 1 and 2, we observed consistent effects in response bias, which suggests that participants are biased to respond "same" when the study and test formats match, but only when a congruency effect is observed (i.e., no bias to respond "same" when both study and test Greebles were aligned in Experiment 1; see Figure 2C). This result suggests that participants are influenced by the similarity in the global configuration of the stimuli. This interpretation of the bias results, the reason that this bias emerged only alongside congruency effects, and whether global configuration also impacts response biases for faces are all questions that remain to be investigated.

Moreover, it has been shown that, with the results from the composite task, we cannot distinguish between holism in the perceptual representation and holism in the decisional process (Richler, Gauthier, et al., 2008). Despite all of these limitations, however, variants of the composite task are widely used in the literature to assess holistic processing (e.g., Diamond \& Carey, 1986; Farah et al., 1998; Gauthier et al., 2003; Goffaux \& Rossion, 2006; Hole, 1994; Hole, George, \& Dunsmore, 1999; Le Grand et al.,
2004; Michel et al., 2006; Robbins \& McKone, 2007; Wenger \& Ingvalson, 2002; Young et al., 1987). Although the pattern of performance in the composite task is characteristic of faces and objects of expertise and can be used to infer holistic processing, one challenge for future work is to disentangle all of these sources of influence in the composite task and to relate them to other face processing paradigms so that we can better understand what makes processing faces and objects of expertise unique.

\section{AUTHOR NOTE}

This research was supported by the James S. McDonnell Foundation, the National Eye Institute, the National Science Foundation (all to I.G.), and by the Temporal Dynamics of Learning Center (NSF Science of Learning Center SBE-0542013). The authors thank Ludvik Bukach, Danielle Brown, and Jim Tanaka for assistance in collecting data, as well as the members of the Perceptual Expertise Network for helpful discussions. Correspondence concerning this article should be sent to J. J. Richler or I. Gauthier, Department of Psychology, Vanderbilt University, Wilson Hall, Nashville, TN 37203 (e-mail: jennifer.j.richler@vanderbilt .edu or isabel.gauthier@vanderbilt.edu).

\section{REFERENCES}

Bentin, S., Sagiv, N., Mecklinger, A., Friederici, A., \& von CraMON, Y. D. (2002). Priming visual face-processing mechanisms: Electrophysiological evidence. Psychological Science, 13, 190-193.

Cheung, O. S., Richler, J. J., Palmeri, T. J., \& Gauthier, I. (2008). Revisiting the role of spatial frequencies in the holistic processing of faces. Journal of Experimental Psychology: Human Perception \& Performance, 34, 1327-1336.

DiAmond, R., \& CAREY, S. (1986). Why faces are and are not special: An effect of expertise. Journal of Experimental Psychology: General, 115, 107-117.

Farah, M. J., Wilson, K. D., Drain, M., \& TAnaKa, J. N. (1998). What is "special" about face perception? Psychological Review, 105, 482498.

Gauthier, I., \& Bukach, C. (2007). Should we reject the expertise hypothesis? Cognition, 103, 322-330.

Gauthier, I., Curran, T., Curby, K. M., \& Collins, D. (2003). Perceptual interference supports a non-modular account of face processing. Nature Neuroscience, 6, 428-432.

Gauthier, I., Klaiman, C., \& Schultz, R. T. (in press). Face composite effects reveal abnormal face processing in autism. Vision Research.

GAUTHIER, I., \& TARR, M. J. (1997). Becoming a "Greeble" expert: Exploring mechanisms for face recognition. Vision Research, 37, 16731682.

GAUThIER, I., \& TARR, M. J. (2002). Unraveling mechanisms for expert object recognition: Bridging brain activity and behavior. Journal of Experimental Psychology: Human Perception \& Performance, 28, 431-446.

Ge, L., Wang, Z., McCleery, J. P., \& Lee, K. (2006). Activation of face expertise and the inversion effect. Psychological Science, 17, 12-16.

Goffaux, V., \& Rossion, B. (2006). Faces are "spatial"-Holistic face perception is supported by low spatial frequencies. Journal of Experimental Psychology: Human Perception \& Performance, 32, 1023-1039.

Hole, G. J. (1994). Configurational factors in the perception of unfamiliar faces. Perception, 23, 65-74.

Hole, G. J., George, P. A., \& Dunsmore, V. (1999). Evidence for holistic processing of faces viewed as photographic negatives. Perception, 28, 341-359

HüBNER, R. (2000). Attention shifting between global and local target levels: The persistence of level-repetition effects. Visual Cognition, 7, 465-484.

Hübner, R., Futterer, T., \& Steinhauser, M. (2001). On attentional control as a source of residual shift costs: Evidence from twocomponent task shifts. Journal of Experimental Psychology: Learning, Memory, \& Cognition, 27, 640-653.

LAWSON, R. (2007). Local and global processing biases fail to influence face, object and word recognition. Visual Cognition, 15, 710-740. 
LEDER, H., \& BRUCE, V. (1998). Local and relational aspects of face distinctiveness. Quarterly Journal of Experimental Psychology: Human Experimental Psychology, 51A, 449-473.

LEDER, H., \& BRUCE, V. (2000). When inverted faces are recognized: The role of configural information in face recognition. Quarterly Journal of Experimental Psychology: Human Experimental Psychology, 53A, 513-536.

Le Grand, R., Mondloch, C. J., Maurer, D., \& Brent, H. P. (2004). Impairment in holistic face processing following early visual deprivation. Psychological Science, 15, 762-768.

MacLEOD, C. M. (1991). Half a century of research on the Stroop effect: An integrative review. Psychological Bulletin, 109, 163-203.

Macmillan, N. A., \& Creelman, C. D. (2005). Detection theory: A user's guide. Mahwah, NJ: Erlbaum.

Macrae, C. N., \& Lewis, H. L. (2002). Do I know you? Processing orientation and face recognition. Psychological Science, 13, 194-196.

Maurer, D., Le Grand, R., \& Mondloch, C. J. (2002). The many faces of configural processing. Trends in Cognitive Science, 6, 255-260.

Michel, C., Rossion, B., Han, J., Chung, C. S., \& Caldara, R. (2006). Holistic processing is finely tuned for faces of one's own race. Psychological Science, 17, 608-615.

Mondloch, C. J., Le Grand, R., \& Maurer, D. (2002). Configural face processing develops more slowly than featural face processing. Perception, 31, 553-566.

NAvon, D. (1977). Forest before trees: The precedence of global features in visual perception. Cognitive Psychology, 9, 353-383.

Perfect, T. J. (2003). Local processing bias impairs line-up performance. Psychological Reports, 93, 393-394.

Richler, J. J., Cheung, O. S., Wong, A. C.-N., \& Gauthier, I. (in press). Does response interference contribute to face composite effects? Psychonomic Bulletin \& Review.

Richler, J. J., Gauthier, I., Wenger, M. J., \& Palmeri, T. J. (2008). Holistic processing of faces: Perceptual and decisional components. Journal of Experimental Psychology: Learning, Memory, \& Cognition, 34, 328-342.

Richler, J. J., TanaKa, J. W., Brown, D. D., \& Gauthier, I. (2008). Why does selective attention to parts fail in face processing? Journal of Experimental Psychology: Learning, Memory, \& Cognition, 34, 1356-1368.

Robbins, R., \& McKone, E. (2007). No face-like processing for objectsof-expertise in three behavioral tasks. Cognition, 103, 34-79.
Rossion, B., KUnG, C.-C., \& TARR, M. J. (2004). Visual expertise with nonface objects leads to competition with the early perceptual processing of faces in the human occipitotemporal cortex. Proceedings of the National Academy of Science, 101, 14521-14526.

SeARCY, J. H., \& Bartlett, J. C. (1996). Inversion and processing of component and spatial-relational information in faces. Journal of Experimental Psychology: Human Perception \& Performance, 22, 904-915.

Sekuler, A. B., Gaspar, C. M., Gold, J. M., \& Bennett, P. J. (2004). Inversion leads to quantitative, not qualitative, changes in face processing. Current Biology, 14, 391-396.

Troje, N., \& BüLthoff, H. H. (1996). Face recognition under varying poses: The role of texture and shape. Vision Research, 36, 1761 -1771 .

Wenger, M. J., \& IngValson, E. M. (2002). A decisional component of holistic encoding. Journal of Experimental Psychology: Learning, Memory, \& Cognition, 28, 872-892.

Weston, N. J., \& Perfect, T. J. (2005). Effects of processing bias on the recognition of composite face halves. Psychonomic Bulletin \& Review, 12, 1038-1042.

Williams, P., \& TARR, M. J. (n.d.). RSVP: Experimental control software for Mac OS 9 [Online]. Retrieved June 22, 1998 from http:// psych.umb.edu/rsvp/.

Wong, A. C.-N., Palmeri, T. J., \& Gauthier, I. (in press). Conditions for face-like expertise with objects: Becoming a Ziggerin expert-but which type? Psychological Science.

YIN, R. K. (1969). Looking at upside-down faces. Journal of Experimental Psychology, 81, 141-145.

Young, A. W., Hellawell, D., \& Hay, D. C. (1987). Configurational information in face perception. Perception, 16, 747-759.

\section{NOTE}

1. Greebles were used in these experiments to avoid any confounds due to uncontrolled previous experience with familiar objects. However, we assume that the pattern of results that we obtained with Greebles in novices are the same as what we would observe using familiar objects, although this remains to be tested.

(Manuscript received February 27, 2008; revision accepted for publication September 26, 2008.) 